\title{
An architecture for integrated crisis management simulation
}

\author{
P. Dihé ${ }^{1}, \underline{\text { R. Denzer }}{ }^{2}$, M. Polese ${ }^{3}$, A.-M. Heikkilä ${ }^{4}$, D. Havlik ${ }^{5}$, \\ J. Sautter ${ }^{6}$, Th. Hell ${ }^{1}$, S. Schlobinski ${ }^{1}$, G. Zuccaro ${ }^{3}$, W. Engelbach ${ }^{6}$ \\ ${ }^{1}$ cismet GmbH, Altenkesseler Str. 17 D2, 66115 Saarbrücken, Germany, \\ ${ }^{2}$ Environmental Informatics Group and cismet GmbH, Goebenstr. 40, 66117 Saarbrücken, Germany \\ ${ }^{3}$ University of Naples Federico II, Via Claudio 21, 80125 Naples, Italy \\ ${ }^{4}$ VTT Technical Research Centre of Finland, Tekniikankatu 1, \\ P.O.Box 1300, FIN-33101 Tampere, Finland \\ ${ }^{5}$ AIT Austrian Institute of Technology GmbH., Donau-City Strasse 1, A1220 Wien, Austria \\ ${ }^{6}$ Fraunhofer-Institut für Arbeitswirtschaft und Organisation, Nobelstr. 12, 70569 Stuttgart, Germany
}

(Email: ralf.denzer@enviromatics.org,ralf.denzer@cismet.de)

\begin{abstract}
The CRISMA project (www.crismaproject.eu ) is a European Union funded project focusing on the simulation of multi-sectoral large scale crisis scenarios that have multi-dimensional effects on society and people. The project aims at the development of a framework to build use case specific tools which will allow decision-makers to cross-examine dynamic crisis scenario evolutions, to set action parameters of operational and strategic activities, and to visualise impacts and crisis evolvement. This will be achieved by providing a modular and open software framework to build planning and decision support systems for modelling and simulating realistic crisis scenarios and their possible consequences.
\end{abstract}

CRISMA applications simulate and analyse the development of a simulated "World" in a crisis management context. A World is defined as a coherent set of data, simulation models operating on this data and the model control parameters governing the activity of these models. A snapshot of the World, or World State, consists of all data related to a specific crisis simulation experiment. This includes a set of information to control simulation models operating on the World data as well as a set of condensed, representative and quantitative information that can be used for a qualitative assessment of a world state. The user can influence a crisis evolvement by changing control parameters of the simulation models. Every modification of a World State is considered a distinct decision point and eventually produces a new World State. This leads to a decision tree.

The architectural design approach uses several concepts from previous projects focusing on reference models, reference architectures and simulation-specific standards. For the CRISMA architecture, the project adopts certain common concepts that support a systematic architectural design process.

In order to demonstrate and validate the design, five pilot sites are used to provide experimentation for validation and testing of a wide range of crisis management situations (coastal floods, extreme weather conditions, geophysical hazards, multi-organisational and cross-border cooperation in crisis management, planning and training for resource management). Piloting will include multi-risk and domino effects.

The paper presents the business logic and key aspects of the CRISMA architecture. The work presented is work in progress, published during the design stage of the architecture. Implementation will be under way during the MODSIM 2013 conference.

Keywords: $\quad$ Planning decision support systems, crisis management simulation, model integration 


\section{INTRODUCTION}

The impact of crisis events is closely related to the capacity of institutions and the public to properly prepare. The simulation of a crisis and possible mitigation activities with the help of mathematical models is a key to the exploration of strategic and operational mitigation options as simulated crisis scenarios can serve as a driver of policy level decision-making instead of simply learning from past experience. The complexity of a crisis situation and the possible severe impacts require multi-sectorial consideration of a crisis instead of focusing on one aspect of the crisis only. However, such consideration is often not achieved, because existing crisis management models and tools operate in a stand-alone fashion (European Commission 2005/European Commission 2012).

Addressing this issue the CRISMA project will provide a modular and open software framework to build planning and decision support systems for modelling and simulating realistic crisis scenarios and their possible consequences. The project thus aims at overcoming the barriers between existing crisis domain specific models and tools with the help of harmonized open interfaces allowing the integration of available and future crisis management models and tools.

CRISMA targets the following types of end users: crisis managers and civil protection practitioners on tactical level, facing logistics, scheduling and management challenges in medium-scale crisis situations; emergency management trainers on tactical level, facing challenges in exercise-support and debriefing; decision makers and consultants on strategic level, facing long-term decisions on resource and infrastructure planning. The CRISMA software framework will support these end users to: a) simulate multi-sectorial crisis scenarios and assess the consequences of an incident, b) simulate possible impacts resulting from alternative actions by simulating consequences of a decision and c) update input for the models (capacities, vulnerability) with real time situational data. Strategic decisions regarding capabilities, related investments, reserves and inventories are supported by assessing available capacities, social vulnerability and resilience of economic systems and impact of hazards on physical infrastructure and environmental assets. User will also be supported in decisions regarding deployment of resources, improvement of action plans for the preparedness and response phases

The envisioned benefits for practitioners in crisis management are: localised decision-support for operations and resource planning; simplified forecasts and what-if-analysis using various simulation models in one software solution; support for training, exercises and planning in resource management, incident evolvement and long-term infrastructure planning.

\section{BUSINESS LOGIC}

CRISMA framework applications simulate and analyse the development of a simulated "World" in a crisis management context. A World is defined as a coherent set of data, simulation models operating on this data and the model control parameters governing the activity of these models. A snapshot of the World, or World State (fig. 1), consists of all data related to a specific crisis simulation experiment. This includes a set of information to control simulation models operating on the World data as well as a set of condensed, representative and quantitative information that can be used for a qualitative assessment of a world state (indicators, and criteria for assessing the meaning of these indicators).

The users can influence crisis evolvement by changing the control parameters of the simulation models. In some applications, the users will be also able to directly change the World State, e.g. by postulating the existence of an infrastructure or introducing additional crisis management resources in the experiment. Every modification of a World State is considered a distinct Decision Point and eventually produces a new World State (World State Transition).

The World State Transition maintains the structure of the World and neither changes the dimension of the World, nor the type of elements that constitute the World State. The actual input and output data for each of the World's simulation models consists of a subset of the elements of the World State. However, the sum of all outputs has to be a full World State to assure comparability of World States. This means that the part of the World State data which is not affected by any of the simulation models needs to be added or referenced in the newly generated World State. 
Dihé et al., An architecture for integrated crisis management simulation

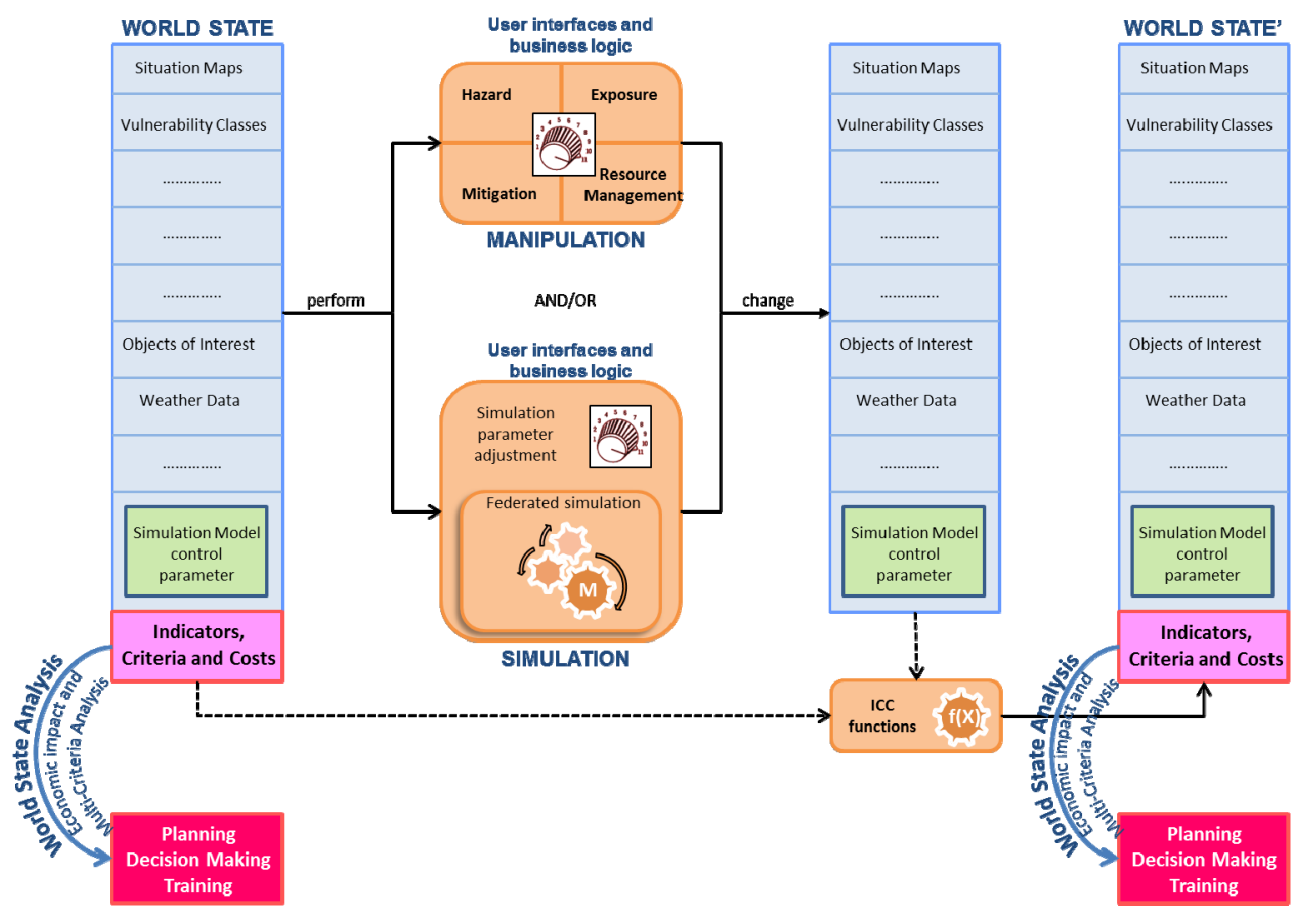

Figure 1. World State Transition and World State Analysis

World State Transitions trigger the production of Indicators, Criteria and Costs (ICC) that are key data representing the world state (e.g. number of dead). ICC data can be considered a less complex aggregation of the World State rather than an integral part thereof. It is used to facilitate the analysis and comparison of World States (e.g. multi-criteria analysis).

CRISMA foresees the possibility of testing and comparing the outcomes of different decisions. This leads to a concept of alternative World States corresponding to alternative decision chains. The alternatives are represented by the set of triples (World State, Transition ${ }^{n},\left\{\right.$ World State', World State,', ..., World State $\left.{ }^{n}\right\}$ ). The logic is independent of crisis management phases and does not explicitly depend on the elapsed time of the simulated crisis. Time-dependency is implicitly included in the World State sequence through scheduling of the Decision Points.

The comparison and analysis of different World States, Alternatives and related Transitions enables crisis managers to explore the effects of changes in hazard, mitigation strategies etc. on the crisis development and final outcomes.

\section{ARCHITECTURE APPROACH}

One major objective of CRISMA is to provide a software framework to build crisis management simulation applications which follow the above pattern of business logic, supporting all dimensions of crisis management simulation (fig. 2). In the following we present goals, design methods, properties and building blocks of the CRISMA Framework Architecture.

\subsection{Architecture Goals}

The CRISMA Framework Architecture has to support integration with existing systems, existing tools, existing models and existing data sources at multiple levels of interoperability. The systems, models, tools and data sources to be integrated in a concrete crisis management application are complex and often not known in advance. This means, that the architecture must be flexible enough to support integration at many levels. In addition, the CRISMA Framework Architecture needs to serve as a blueprint for concrete Application Architectures of the crisis management domain. As a consequence, the CRISMA Framework Architecture is split in two parts. The Functional Architecture is concerned with the general concepts, relationships and functional Building Blocks independent of aspects referring to the realisation of a specific crisis management simulation application. The Implementation Architecture provides a concrete architecture of a specific crisis management simulation application in terms of software components and information 
models. The Functional Architecture has to ensure composability and reusability of Building Blocks, especially in the context of integration into external systems. Moreover, the Functional Architecture has to select consistent standards and specifications for the exchange of information between components.

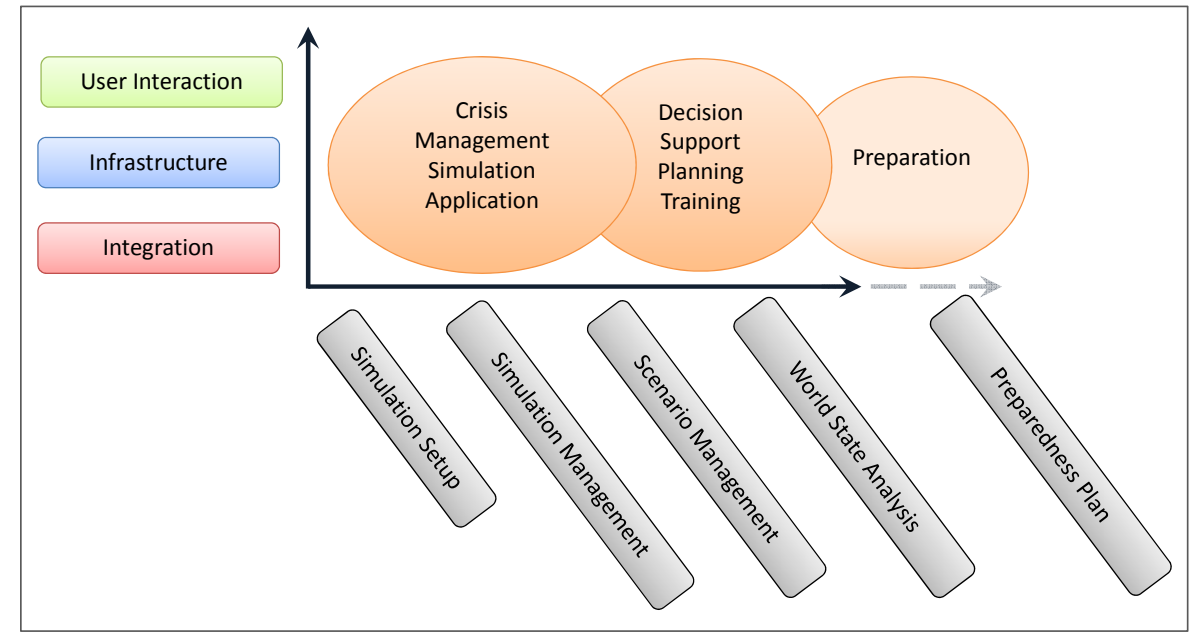

Figure 2. CRISMA Framework Dimensions

\subsection{Architecture Specification}

The architecture specification is structured along four main viewpoints according to the MCRI pyramid: Mission, Concepts, Realisation and Implementation (Rome 2009). The mission viewpoint defines the primary goals of the CRISMA Framework and documents how the architectural goals, properties and constraints have been derived from project objectives and user requirements. The concepts viewpoint defines the key concepts that are applied to realise the goals. They encompass general architectural concepts like reference architecture, Building Blocks, integration concepts and user oriented concepts for resource management, training and so on. The realisation viewpoint addresses the realisation of the concepts by a set of functional Building Blocks and their interaction patterns. Functional Building Blocks provide an abstract description of the general functionalities to be provided by the CRISMA Framework. The implementation viewpoint further decomposes the functional Building Blocks into concrete software components.

\subsection{Architecture Design Approach}

The architectural design approach uses several concepts from previous projects focusing on reference models, reference architectures and simulation-specific standards. The approach taken in CRISMA does not directly adopt a certain architecture or reference model, like RM-ODP (RM-ODP 1998), RM-OA (RM-OA, 2007), SOA-RM (SOA-RM, 2006) or TOGAF (TOGAF, 2013). Nevertheless, the project adopts certain common concepts that support a systematic architectural design process:

- The four layer MCRI scheme (Mission, Concepts, Realisation, Implementation) is used to provide the overall structure of the CRISMA Framework Architecture (Rome, 2009).

- Overall architectural goals, properties and constraints are identified to guide the architectural development.

- The architecture is separated into an implementation independent (Functional Architecture) and implementation dependent (Implementation Architecture / Application Architecture(s)), based on concepts introduced in the Reference Model for the ORCHESTRA Architecture (Usländer 2010).

- The architecture defines the framework that provides specific functionality in the form of Building Blocks. Functional Building Blocks are identified and decomposed in a stepwise fashion into a hierarchical set of components (tools, services) with well-defined interfaces and interaction concepts.

- Moreover, a methodology and rules for data, model and application integration are defined and respective tools and standards for data and model integration are specified. 


\subsection{CRISMA Building Blocks}

The CRISMA Framework Architecture defines three types of Building Blocks: 1.) User Interaction; 2.) Infrastructure; 3.) Integration. At the heart of the framework are middleware components responsible for the management of world state data and simulations. The framework middleware components support common functionality like access control, search and discovery.

As shown in Fig. 3 Building Block functionality includes the support for the integration of data and simulation models, simulation and scenario management. Building Blocks for world state analysis as well as the creation of preparedness plans are foreseen for the next version of the Framework to be finalised in the course of 2014.
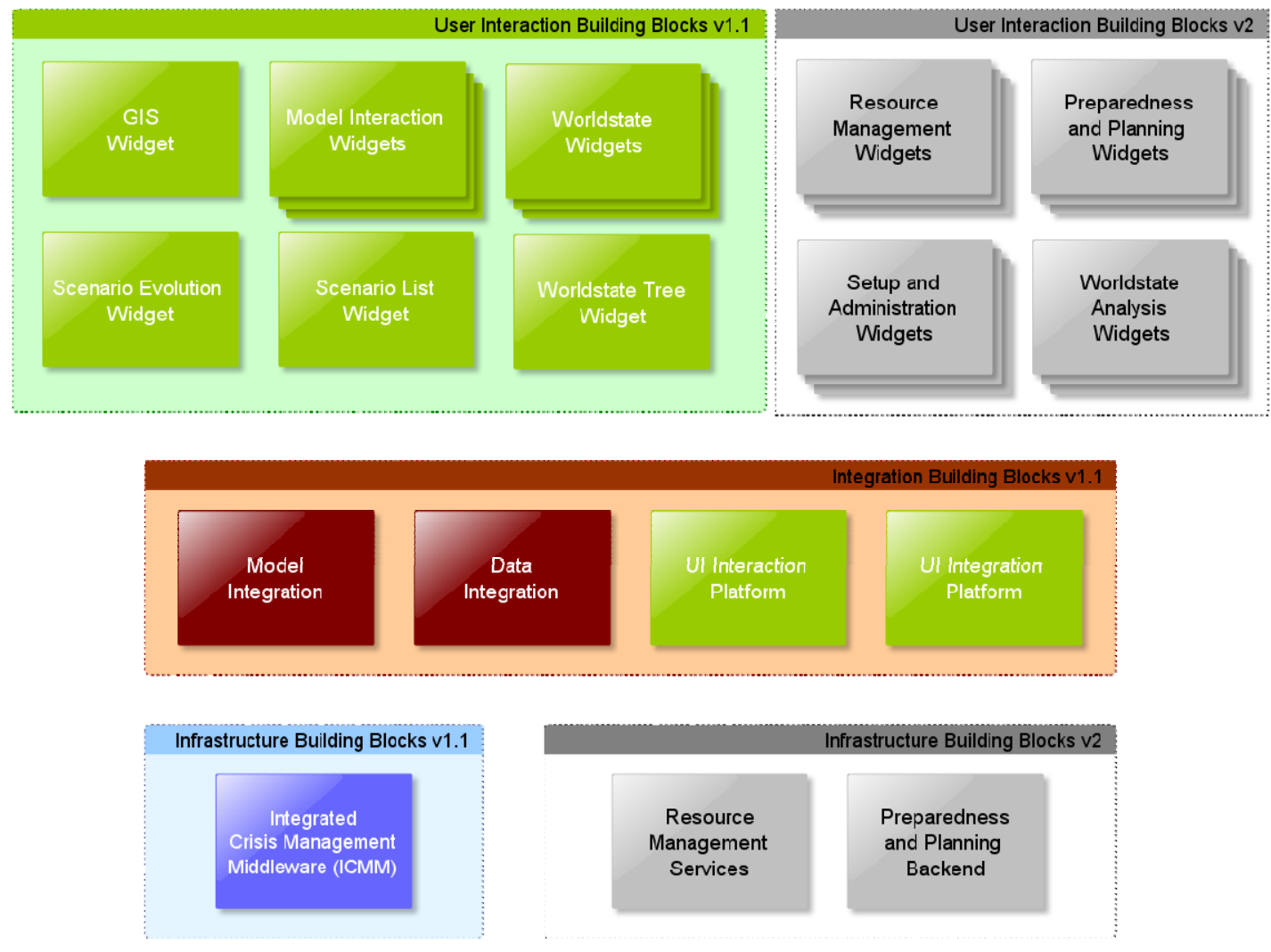

Figure 3. CRISMA Framework Building Blocks

In addition to the integration at service level, the CRISMA Framework Architecture foresees integration at GUI level, by means of functional GUI widgets (Composite UI Modules) linked to other Building Blocks. These widgets can be combined in ad-hoc web mashup applications as needed. The result can be either used stand-alone or embedded in existing applications.

\subsection{End user perspective}

CRISMA end-users work with predefined Simulation Cases that are packages of models, data, pre-set parameters and parameters that can be changed during the training or planning exercise (Simulated Crisis Scenario). The Simulation Cases are supported by a set of Building Blocks. Fig. 4 shows the use of Building Blocks in the context of a possible specific crisis management simulation application. The CRISMA project endorses a number of so-called "reference applications" that serve to demonstrate the concepts of the framework and that can be used as examples of possible CRISMA applications.

World states represent snapshots of the world generated by simulation models or by manual user inputs (e.g. simple definition of impacts scenario by positioning of casualties). In terms of the technical concept world states are not separated in crisis evolvement phases (incident/response/restore) as it is not needed 
conceptually. However, for an end-user interacting with a CRISMA application it is crucial to clearly distinguish whether we deal with a situation caused by an incident (impact) or with a situation that reflects the operational picture of rescue/response resources (ambulances, fire trucks etc). According to the CRISMA interaction concept an end-user can deal with a surrounding world scenario (properties of the world not directly affected by the crisis), an impact scenario (harms, casualties etc.) and with a response scenario (fire trucks, ambulances etc.) all represented using the architecture concept world state.

The "decision making" related to possible "what if"-scenarios corresponds to sets of changeable parameters exposed by a Simulation Case. This way experiments can be performed at specific Decision Points represented by a set of world state data. In a World State Tree users are enabled to navigate a simulated crisis scenario (step by step over time), explore different mitigation options (World State Widgets) and eventually compare the results of different previously taken decisions. The CRISMA Framework provides a mean to keep track and compare "alternative decision scenarios" resulting from such related decision trees.

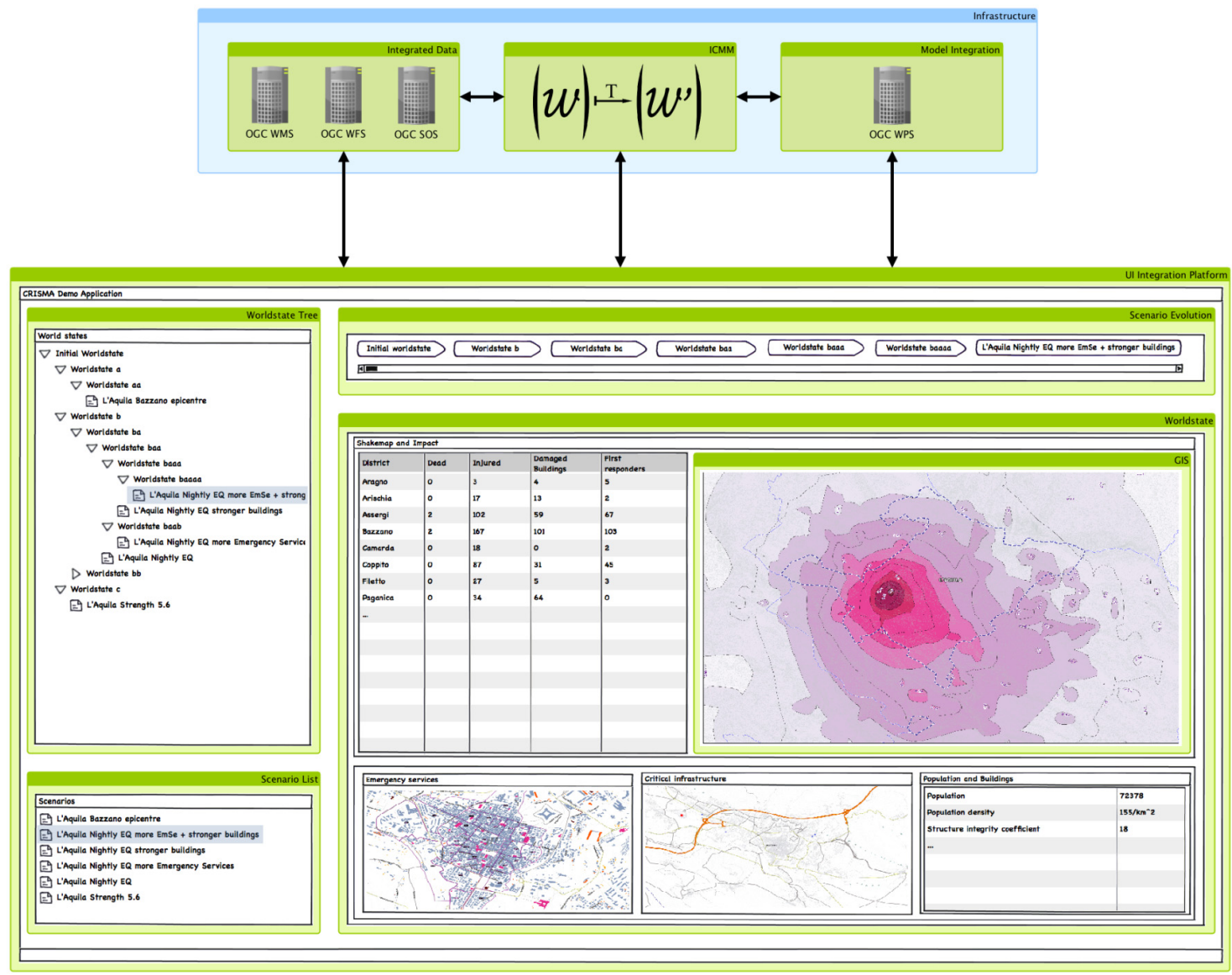

Figure 4. CRISMA Framework Reference Application

\section{DEMONSTRATIONS}

In order to demonstrate and validate the CRISMA results, five pilot sites are used to provide an experimentation frame for validation and testing by behalf of a wide range of crisis management situations including multi-risk and domino effects. These pilots are in several usage contexts focused around the following risk domains and crisis management topics:

- Coastal floods and other extreme weather conditions

- Geophysical hazards

- Multi-organisational and cross-border cooperation in crisis management

- Planning and training for resource management 
Dihé et al., An architecture for integrated crisis management simulation

Piloting will commence in the third quarter of 2013.

\section{ACKNOWLEDGEMENTS}

CRISMA is funded by the European Community's 7th Framework Programme under the grant agreement no. 284552.

\section{REFERENCES}

Denzer R. (2002). Generic integration of environmental decision support systems - state-of-the-art, Environmental Modelling \& Software, Volume 20, Issue 10, pp. 1217-1223.

Denzer, R., Schlobinski S., Gidhagen L. (2011). A Decision Support System for Urban Climate Change Adaptation, In: Proceedings of the 44th Hawaii International Conference on System Sciences (HICSS44), CDROM, IEEE Computer Society.

European Commission (2005) Towards an open disaster risk management service architecture for INSPIRE and GMES. White Paper.

European Commission (2012) What is the Shared Environmental Information System? [http://ec.europa.eu/environment/seis/what.htm]

SOA-RM, (2006), https://www.oasis-open.org/committees/tc_home.php?wg_abbrev=soa-rm

RM-OA, Usländer Th. (ed.) (2007): Reference Model for the ORCHESTRA Architecture (RM-OA Version 2.1), OGC Best Practices Document, Open Geospatial Consortium, portal.opengeospatial.org/files/?artifact_id=23286

RM-ODP, ISO/IEC 10746 Reference Model for Open Distributed Processing, 1996 and 1998.

Rome, E., Bologna, S., Gelenbe, E., Luiijf, E., Masucci, V. (2009): DIESIS: An Interoperable European Federated Simulation Network for Critical Infrastructures. In Proceedings of the 2009 SISO European Simulation Interoperability Workshop (ESIW'09), Istanbul, Turkey, July 13-16, pp. 139-146. San Diego, CA: Simulation Councils, Inc.

TOGAF, http://www.opengroup.org/togaf/

Usländer Th., Denzer R., Requirements and Open Architecture of Environmental Risk Management Information Systems, in: B. van der Walle (ed.), Information Systems for Emergency Management, M. E. Sharpe Publishers, ISBN 978-0-7656-2134-4, pp. 344-368, 2010 